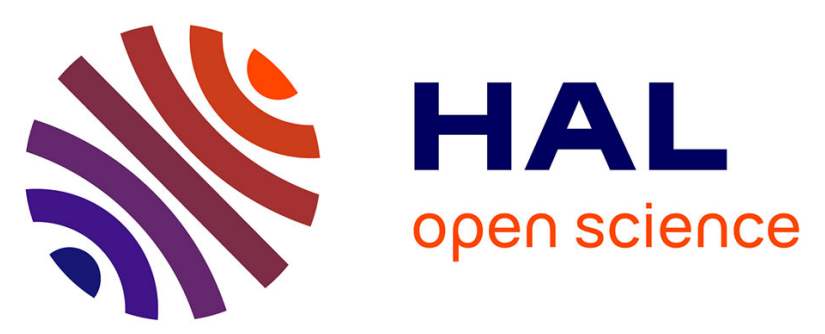

\title{
Identification of acquisition parameters from the point spread function of a fluorescence microscope
}

Olivier Haeberlé, F. Bicha, Christophe Simler, Alain Dieterlen, Chengqi Xu, Bruno Colicchio, Serge Jacquey, Marie-Pierre Gramain

\section{- To cite this version:}

Olivier Haeberlé, F. Bicha, Christophe Simler, Alain Dieterlen, Chengqi Xu, et al.. Identification of acquisition parameters from the point spread function of a fluorescence microscope. Optics Communications, 2001, 196, pp.109-117. 10.1016/S0030-4018(01)01376-1 . hal-00954949

\section{HAL Id: hal-00954949 \\ https://hal.science/hal-00954949}

Submitted on 3 Mar 2014

HAL is a multi-disciplinary open access archive for the deposit and dissemination of scientific research documents, whether they are published or not. The documents may come from teaching and research institutions in France or abroad, or from public or private research centers.
L'archive ouverte pluridisciplinaire HAL, est destinée au dépôt et à la diffusion de documents scientifiques de niveau recherche, publiés ou non, émanant des établissements d'enseignement et de recherche français ou étrangers, des laboratoires publics ou privés. 


\title{
Identification of acquisition parameters from the point spread function of a fluorescence microscope
}

\author{
O. Haeberlé, F. Bicha, C. Simler, A. Dieterlen, C. Xu, B. Colicchio and S. Jacquey \\ Groupe Lab.El - Equipe MIPS, Université de Haute-Alsace \\ IUT Mulhouse, 61 rue A. Camus F-68093 Mulhouse Cedex France \\ M.-P. Grammain \\ Laboratoire de Recherche en Instrumentation Médicale Automatisée en Cancérologie \\ (IMAC, CRAN URA CNRS D0821) \\ Laboratoire de Recherche en Oncologie (LRO), Centre Alexis Vautrin \\ Avenue de Bourgogne, F-54511 Vandouvre-Les-Nancy, France
}

\begin{abstract}
:
Except for blind methods, deconvolution of 3-D data sets acquired from a fluorescence microscope requires the knowledge of the point spread function (PSF) of the instrument. Using the XCOSM package, we show first with simulations and then with recorded data that it is possible to recover from an experimental PSF some parameters, which are very difficult or impossible to measure during the acquisition, like the specimen depth or the immersion medium refractive index. Doing so, we can precise the acquisition protocol, which helps to use the instrument under optimal conditions. Furthermore, the knowledge of the actual acquisition conditions permits to use for the deconvolution process a computed PSF, which is noiseless and as close as possible to the actual PSF. This helps to reduce errors in quantitative measurements after deconvolution, as shown with computations.
\end{abstract}

Keywords: point spread function, microscopy, deconvolution, system identification 


\section{INTRODUCTION}

Fluorescence microscopy is a key tool to study three-dimensional (3-D) structures of living cells and tissues [1-3]. Recent developments in instrumentation have permitted to overcome the conventional resolution limit with the inventions of the confocal, multi-photon and 4-pi microscopes for example [4-6]. New techniques promise to push the limit even further [7].

When the ultimate resolution is obtained from the instrument, it is still possible to sharpen the images by deconvolution of the data using the instrument point spread function. Each recorded image $g(X)$ can be described by the following convolution equation:

$$
g(X)=\int_{-\infty}^{+\infty} h\left(X-X_{1}\right) f\left(X_{1}\right) d X_{1} \oplus b(X)
$$

where $\mathrm{X}$ and $\mathrm{X}_{1}$ are 3-D coordinates $\mathrm{h}(\mathrm{X})$ is the Point Spread Function (PSF) of the acquisition system and $f(X)$ is the original object. The term $b(X)$ represents a random process. It is a combination of noise sources due to the fluorescence process and the acquisition electronics.

Deconvolution consists in inverting Eq. (1) in order to find an estimate of the actual object $f(X)$, knowing the image $\mathrm{g}(\mathrm{X})$ and the point spread function $\mathrm{h}(\mathrm{X})$. Deconvolution is known to be an ill-posed problem, the process being sensitive to initial conditions and noise. Furthermore, it can be very time consuming, especially for 3-D data sets. A large amount of effort has been devoted to find new or improve existing deconvolution algorithms (Refs. [8-14] and references therein).

The PSF of the microscope can be measured, usually by acquiring the image of small fluorescent latex beads, ideally of much smaller size than the instrument resolution. Experimental PSFs are however noisy, which can constitute a limitation for the deconvolution process (catastrophic amplification of the noise). Furthermore, the use of very small beads leads to bad signal to noise ratio, so that one often uses beads of size not being much smaller than but of the order of the microscope resolution. As a consequence, the size of the PSF may be overestimated. On the other hand, doing so ensures that the recorded PSF takes into account the full acquisition chain, and all its possible defects.

Computed PSFs are noiseless, but do rely on a simplified model of the microscope. Furthermore, the assumed experimental parameters, which are used in the computational model, may differ from their actual values. The PSF is very sensitive to some parameters like the immersion oil index, the depth of the specimen under the coverslip, or the numerical aperture of the objective. Figure 1(a) shows a noisy experimental PSF: the slight asymmetry indicates that at least one parameter differs from the manufacturer's recommendation, inducing aberrations. Figure 1(b) shows a theoretical PSF computed assuming design parameters only: deconvolving an image with a computed PSF which differs from the actual experimental PSF corresponding to the specimen image acquisition may lead to large errors in the final result.

We investigate the possibility to recover some parameters which are difficult or impossible to measure directly (like the specimen depth under the coverslip) from a recorded PSF. Doing so, a computed PSF which is noiseless and as close as possible to the actual PSF can be used for the deconvolution. Identifying experimental parameters should also help to characterize the experimental protocol, an important point for the biologists. 


\section{METHOD}

The method to extract experimental parameters from a recorded PSF is the following: varying one parameter in a physically realistic interval, one computes PSFs, which are compared to a reference PSF (see Fig. 2). Both the reference and the computed PSFs are normalized, and the correlation coefficient and the quadratic error are chosen as comparators. Finally, one gets the value of the parameter for which the best resemblance is obtained. The same technique can be extended if several parameters are to be found.

It is worth noting some limitations inherent to the method, due to the way of measuring a PSF: recording the 3-D image of a fluorescent specimen or of a small bead to acquire a PSF is done by optical sectioning. The focal plane is scanned through the specimen, and at each position a 2-D picture is taken. Reconstruction is done with a computer.

Two difficulties appear: measuring its PSF is insufficient to fully characterize the optical system: one records only an intensity information whereas the phase information is lost. Knowledge of the phase is mandatory to quantify the aberrations. Consequently, identifying one or several parameters is not a characterization of the optical quality of the system.

A second limitation of the technique is the miss of an absolute reference frame: when recording a PSF, one looks for the maximum intensity plane which is taken as a reference, and one takes a set of images in planes above and below the central plane of maximum intensity. An experimental PSF is therefore automatically centered in its domain of acquisition. When computing a PSF, one uses an absolute reference frame centered at the geometrical position of the focal point $[19,25,27]$. The distance between this theoretical position and the plane of maximum intensity is in practice unknown, and this represents also a loss of information. It is therefore necessary to calculate this focal shift in order to center the computed PSF (Fig. 2).

As a consequence, one could argue if the found solution is unique, and if it is correct. In order to test the method, we first use as a reference PSF a centered computed PSF, to which we add Poissonian noise to mimic the fluorescence phenomenon [15] and Gaussian noise to take into account the contribution of the acquisition system electronics.

\section{CHOICE OF A MICROSCOPE MODEL}

The description of waves in focal regions has lead to numerous efforts from many authors and the microscope in particular has generated intensive work to establish theoretical models of the image formation process using diffraction theories [16-28].

For high numerical aperture objectives, the extremal incident rays are impinging at large angles of incidence on the various interfaces separating the microscope objective from the specimen and as a consequence, vectorial theories of diffraction seem mandatory. However, "Gibson and Lanni demonstrated good agreements between their numerical results and experimental measurements of the aberrated point spread function $[26,27]$. This and some other theories confirm that, while it is essential to construct mathematically rigorous theories, it is sometimes possible to obtain accurate predictions using approximate physical models based on wave optics" (from Ref. [28]). 
Therefore we decide to use this approximate physical model as implemented in the XCOSM package [29]. This software from the Biomedical Computer Laboratory (Washington University, St Louis, Missouri, USA) provides the implementation of several algorithms for deconvolving 3-D images, as well as for computing point spread functions from optical or confocal microscopes. It runs on Unix workstations and PCs. From this package, we use to compute PSFs a single routine xcosm_psf, which can be called independently.

The Gibson and Lanni model [26,27] introduces eight parameters (out of a total of 18 parameters, see Annex) which may vary from their design conditions as recommended by the microscope manufacturer. These eight parameters are:

- the specimen index and its depth under the coverslip

- the thickness and index of refraction of the immersion medium

- the numerical aperture of the objective

- the thickness and index of refraction of the coverslip

- the tube length

Out of these eight parameters, we choose to focus our attention on three parameters that are difficult or impossible to measure in practice:

- The depth of the specimen under the coverslip is in practice impossible to measure directly. When using fluorescent spheres in water, useful beads are those sticking to the coverslip after being dried on it. Those floating into the water layer are affected by brownian motion and therefore cannot be used to measure a PSF. A solution is to embed the beads in a gel with a refractive index close to water [27].

- The oil refractive index is sensitive to temperature, and illumination of the sample is susceptible to heat the immersion medium. Also, the value is often measured near $\mathrm{T}=20^{\circ} \mathrm{C}$ by the manufacturer [30], while some biologists do work with living cells at $\mathrm{T}=37^{\circ} \mathrm{C}$, the microscope staying in a temperature controlled enclosure. This may induce on this value an error of $4 \%$ only, but the PSF is very sensitive to this parameter [27].

- Finally, the numerical aperture of the objective may be limited to lower than indicated values. For example, when focusing into a watery medium, the numerical aperture is at best 1.33 , because total internal reflections limit the collecting angle.

Using the original XCOSM package, the PSF is computed in a volume centered around the theoretical focal point when all design parameters are used. When non-design parameters are used, the PSF may be shifted with respect to this theoretical focal point. The problem of the focal shift is then handled as follows: the focal shift is calculated using the original xcosm_psf routine [29], the point spread function being computed along the optical axis only using a very small z-scanning step to accurately determine the shift. We have recompiled the xcosm_psf routine and added the focal shift as a new parameter in the template file. Then, the recompiled routine xcosm_psf_shift is used with the actual z-scanning step to compute a PSF, which is correctly centered in its domain of definition. 


\section{TESTS ON COMPUTED PSFS}

We first focus our attention on the identification of one parameter only. A PSF has been calculated with an objective of numerical aperture (NA) 1.26, using an immersion oil of actual index (medacti) 1.513 for a specimen depth (specthick) of $2 \mu \mathrm{m}$. All other parameters correspond to the design conditions. The annex describes the various parameters of the Gibson and Lanni model, and gives the values, which have been used for computations. Poissonian noise was then added to the reference PSF to simulate the fluorescence process. Gaussian noise (Signal to Noise ratio of $40 \mathrm{~dB}$ ) was further added to take into account electronic noise. The level of $40 \mathrm{~dB}$ was chosen after analysis of actual images from a 3-D fluorescence microscope.

Then one tries to find these parameters by comparing PSFs computed with variable NA, medacti or specthick. The variation interval for each parameter was set to $1.20 \leq \mathrm{NA} \leq 1.30$ in steps of 0.01 , $1.510 \leq$ medacti $\leq 1.520$ in steps of 0.001 and $0 \mu \mathrm{m} \leq$ specthick $\leq 3 \mu \mathrm{m}$ in steps of $0.1 \mu \mathrm{m}$.

When directly using the images blurred with Gaussian and Poissonian noise, the obtained results are sometimes useless. In order to improve the procedure, a simple denoising procedure is used, consisting in evaluating the noise mean value on one plane of the image (in a dark region of the PSF) and clipping the mean value to the entire 3-D image [31]. The curves presented here are all obtained for deblurred images. Fig. 3(a), (b) and (c) show the results using the correlation coefficient as comparator. The oil immersion index is found correctly at 1.513 . The numerical aperture is found with a very small error at $N A=1.27$. The depth of the specimen if found with a larger, $10 \%$ error at $1.8 \mu \mathrm{m}$ instead of $2 \mu \mathrm{m}$.

Repeating the computations for several values of these parameters has shown that the numerical aperture is found with an error smaller than $1 \%$ when the depth of the specimen below the coverslip is less than $5 \mu \mathrm{m}$. The immersion oil refractive index is found with a smaller error, usually of the order of $2 \%$. The depth of the specimen is found with a larger error of $10-15 \%$.

However, the results degrade very rapidly when the level of noise increases. For a signal to noise level lower than $25 \mathrm{~dB}$, it was not possible to find the unknown parameters with any reasonable precision. Same results are obtained when using the quadratic error as comparator between the reference PSF and the computed ones.

It is worth noting that better results are obtained when the unknown parameter does in fact not induce a focal shift, as for the numerical aperture. The correlation curve is very smooth. When there is a focal shift due to the use of a non-design parameter (specimen depth for example), the centering of the reference and computed PSFs in their domain of definition is very important. Even small errors in the focal shift may induce irregularities in the correlation curve (see Fig. 3(c)).

When two or more unknown parameters have to be found, the problem of local mimima arises. We found that when for physical reasons the variation intervals may be restricted enough, no local minimum occurs.

The precision is however often much lower. Table 1 shows the results we obtained for a two-parameter search (immersion medium refractive index and depth under the coverslip) for a water immersion objective. The reference PSF is computed using the Gibson and Lanni model, blurred by the addition of Poissonian and Gaussian noise, and denoised with the clipping method [31]. While the immersion refractive index of water is found with a fairly good precision (4\%o), a larger error ( $>40 \%)$ occurs on the specimen depth. 


\section{EXPERIMENTAL PSFS}

The results we obtained by simulations encouraged us to test our method on experimental PSFs acquired on an AX70 Provis epifluorescence microscope (Olympus), equipped with a CellScan 3-D acquisition device. Images are acquired using a 12 bit, Sensys CCD camera (Photometrics). Pixel size is $6.8 \times 6.8 \mu \mathrm{m}^{2}$. TetraSpeck fluorescent latex beads of $0.2 \mu \mathrm{m}$ diameter have been used (Molecular Probes). The beads have been dried on a coverslip. Then the coverslip is sealed onto a glass slide with a small layer of water in between. Doing so, some beads do not stick anymore to the coverslip, and float in the watery medium. They are then affected by brownian motion, and cannot be used to acquire PSFs. What may be a drawback ensures in our measurements that only PSFs at a null depth under the coverslip are acquired, in a 1.33 refractive index medium. A 100x, oil immersion (medacti=medesri=1.515), NA=1.35 objective has been chosen, and coverslips with design parameters have been used (see Annex).

The images we obtained were denoised the same way as the computed reference PSFs. We then verified that the correct specimen depth and oil refractive index are found, as shown on Fig. 4(a) and (b). These two curves represent the quadratic error between the experimental reference PSF and the computed PSFs: the minima occur at a specimen depth of $0 \mu \mathrm{m}$ (which is the expected value) and for an immersion oil refractive index of 1.516. This latter value is only very slightly different from the manufacturer's data [30], the difference being in the error range of our method. Same results were obtained when using the correlation coefficient.

\section{IMPORTANCE FOR DECONVOLUTION}

As mentioned in the introduction, the knowledge of exact acquisition parameters is important when using a noise free computed PSF for deconvolving images. The shape of the PSF is for example very sensitive with respect to the oil refractive index [27]. To study its influence on the deconvolution result, we have simulated the image of a $680 \mathrm{~nm}$ diameter fluorescent bead acquired at $37^{\circ} \mathrm{C}$ (as when using the microscope for studying living cells), taking into account the temperature correction given by the oil manufacturer [30] with respect to the value of 1.516 we have found when analyzing our experimental PSF acquired at $21^{\circ} \mathrm{C}$. The actual $\mathrm{PSF}_{1.512}$ must then be computed with an oil refractive index of 1.512. The $680 \mathrm{~nm}$ diameter solid sphere is first convolved with $\mathrm{PSF}_{1.512}$. The obtained simulated image is blurred with Poissonian and Gaussian noise as explained previously. It is then deconvolved, on the one hand using $\mathrm{PSF}_{1.512}$ computed at $37{ }^{\circ} \mathrm{C}$ and on the other hand using $\mathrm{PSF}_{1.515}$ computed with an incorrect index of 1.515 corresponding to the manufacturer's recommendations.

Figure 5 illustrates the results we obtained when deconvolving the image using the Linear Least squares Solution (LLS) algorithm [32-33]. Figure 5(a) shows the bead. Figure 5(b) represents PSF ${ }_{1.512}$ : note the asymmetry due to the non-design value of 1.512 for the immersion oil (design value of 1.515) because of the change in temperature. Figure 5(c) is the simulated image resulting from the convolution of the bead with PSF $_{1.512}$. Figure 5(d) and 5(e) show the result of the LLS deconvolution with PSF PS1. $_{12}$ and $\mathrm{PSF}_{1.515}$ (shown on Fig. 5(f)), respectively. Note that the shape of the original object is better reconstructed when using the correct $\mathrm{PSF}_{1.512}$, as almost no asymmetry remains. 
Compared with the original bead, a $15 \%$ level segmentation on the undeconvolved image gives an error on volume measurement of $692 \%$, mostly because of the important z-elongation. This shows that volumic measurements on raw data acquired with an optical microscope are irrelevant. When using the correct $\mathrm{PSF}_{1.512}$ this error is still of $56 \%$ (because the LLS algorithm does not fully reconstruct the original shape), but when using $\mathrm{PSF}_{1.515}$, the error is larger at $91 \%$. This shows that a supplemental error of $25 \%$ in the size measurement is done when compared to the image deconvolved with the correct PSF.

\section{CONCLUSION AND PERSPECTIVES}

We have shown that it is possible to extract from a 3-D computed PSF some parameters used in the Gibson and Lanni model. Then, we used experimental PSFs to confirm that some parameters of the acquisition system, like the index of refraction of the immersion medium or the depth of the fluorescent bead under the coverslip do actually have the expected values.

The precision of the obtained results is however strongly limited by the level of noise in the image which must be kept as low as possible. The acquisition precision is also very important: the z-scanning steps must be as small as possible in order to obtain a good definition along the optical axis, and the centering of the experimental PSF in its domain of definition is crucial. This is probably the most severe limitation of the method.

Among possible improvements is the use of better PSF denoising algorithms, which should help working on lower quality, noisier images. More elaborate comparison criteria could also be used, which should lower the importance of the centering of the PSF in its domain of definition. This centering is easy to obtain laterally, but quite difficult along the optical axis because of the much cruder sampling. A probably more accurate vectorial model for the microscope image formation process can also be used. In order to routinely use this method of identification, a speed bump is mandatory: a one-parameter search takes usually less than $10 \mathrm{mn}$ on a DEC $500 \mathrm{MHz}$ Alpha workstation, but a two-parameter research may take several hours. When the unicity of the solution is expected, one could use extremum research algorithms, which would greatly accelerate the process.

The proposed technique permits to use computed PSFs which are as close as possible to experimental ones. Being noiseless and not relying on theoretical design values, they are more suited for deconvolving 3D data sets. In a future work, we plan to quantify the gain obtained by using optimal computed PSFs versus experimental PSFs. We also plan to use fluorescent beads embedded at a precisely known depth in a well characterized (refractive index) gel matrix to explore the domain of usefulness of the method, in view of improving the deconvolution of images of thick specimen.

This method should also permit to identify experimental parameters, which are in practice difficult to measure, and therefore may help biologists to improve the acquisition protocol.

Finally, the method may also be used for confocal microscopes. 


\section{ANNEX}

List of the parameters used to compute the PSF of an optical microscope with the XCOSM package:

Nxy: 128

size of the image in $\mathrm{x}$ and $\mathrm{y}$

deltaxy: 0.068

pixel size in image space in $\mu \mathrm{m}$

Nz: 128

size of the image in $\mathrm{z}$ (optical axis)

deltaz: 0.1

pixel size in $\mathrm{z}$ in $\mu \mathrm{m}$

mag: 100

lateral magnification

NA: $1.26^{*}$

numerical aperture of the objective

workdist: 0.16

working distance of the objective in $\mathrm{mm}$

lamda: 0.000530

fluorescence wavelength in $\mathrm{mm}$

slipdesri: 1.522

coverslip design refractive index

slipactri: 1.522

coverslip actual refractive index

slipdesth: 0.170

coverslip design thickness in $\mathrm{mm}$

slipactth: 0.170

coverslip actual thickness in $\mathrm{mm}$

medesri: 1.515

medactri: $1.513^{*}$

specri: 1.33

specthick: $0.0^{*}$

desot: 160

immersion oil design refractive index

immersion oil actual refractive index

specimen refractive index

specimen depth in $\mathrm{mm}$

design tube length in $\mathrm{mm}$

actot: 160

actual tube length in $\mathrm{mm}$

focalshift: $0^{* *} \quad$ focal shift if non-design conditions $(\mu \mathrm{m})$

* variables to which we focus our attention and which vary during the computations.

** focal shift used to compute a PSF centered in its domain of definition with our recompiled xcosm_psf_shift routine. 


\section{REFERENCES}

[1] T. Wilson and C.J.R. Sheppard "Theory and practice of Scanning Optical Microscopy" Academic Press, London (1984)

[2] D.A. Agard, Y. Hiraoka, P. Shaw and J.W. Sedat in "Methods in Cell Biology, Fluorescence Microscopy in Three-Dimensions Vol.30" p. 353, D.L. Taylor and Y. Wang eds., Academic, San Diego, Calif. (1989)

[3] S. Hell, E. Lehtonen and E.H.K. Stelzer, in "New Dimensions of Visualization in Biomedical Microscopies", p. 145, A. Kritte ed., Verlag Chemie, Weinheim, Germany (1992)

[4] D. Minsky, Scanning 10, p. 128 (1988)

[5] W. Denk, J.H. Strickler and W.W. Webb, Science 248, p. 73 (1990)

[6] S. Hell and E.H.K. Stelzer, Opt. Comm. 93, p.277 (1992)

[7] T.A. Klar and S.W. Hell, Opt. Lett. 24, p. 954 (1999)

[8] B.R. Hunt, Inter. J. of Mod. Phys. C 5, p. 151 (1994)

[9] T.J. Holmes, J. Opt. Soc. Am A 8, p. 893 (1991)

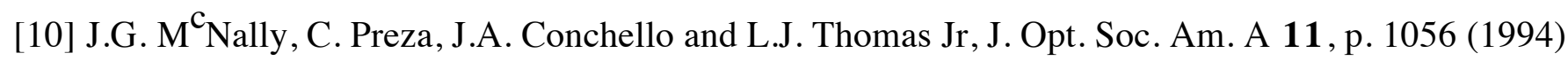

[11] J.A. Conchello, J.J. Kim and E.W. Hansen, App. Opt. 33, p. 3740 (1994)

[12] J.A. Conchello, SPIE 2302, p. 369 (1994)

[13] J.A. Conchello, J. Opt. Soc. Am. A 15, p. 2609 (1998)

[14] P.J. Verveer and T.M. Jovin, J. Opt. Soc. Am. A 14, p. 1696 (1997)

[15] D. Axelrod, Biophys. J. 26, p. 557 (1979)

[16] J.J. Stamnes "Waves in focal regions" Adam Hilger, Bristol (1986)

[17] C.J.R. Sheppard and C.J. Cogswell, Optik 87, p.34 (1991)

[18] C.J.R. Sheppard, M. Gu and K. Brain, Appl. Opt. 33, p.616 (1994)

[19] C.J.R. Sheppard and P. Török, J. Microsc. 185, p.366 (1997)

[20] P. Török, P. Varga, Z. Laczik and G.R. Booker, J. Opt. Soc. Am. A 12, p. 325 (1995)

[21] P. Török, P. Varga and G.R. Booker, J. Opt. Soc. Am. A 12, p. 2136 (1995)

[22] P. Török, P. Varga, A. Konkol and G.R. Booker J. Opt. Soc. Am. A 13, p. 2232 (1996)

[23] P. Török and P. Varga, Appl. Opt. 36, p.2305 (1997)

[24] S.H. Wiersma and T.D. Visser, J. Opt. Soc. Am. A 13, p. 320 (1996)

[25] S. Hell, G. Reiner, C. Cremer and E.H.K. Stelzer, J. Microsc. 169, p. 391 (1993)

[26] S.F. Gibson and F. Lanni, J. Opt. Soc. Am. A 6, p. 1357 (1989)

[27] S.F. Gibson and F. Lanni, J. Opt. Soc. Am. A 8, p. 1601 (1991)

[28] P. Török, S.J. Hewlett and P. Varga, J. Microsc. 188, p. 158 (1997)

[29] www.ibc.wustl.edu/bcl/xcosm/xcosm.html

[30] the index of refraction of the oil we used was given by the manufacturer (Cargille) as $n=1.515 \pm 0.002$ at $\mathrm{T}=23^{\circ} \mathrm{C}$ with a temperature correction coefficient of $-0.00031 / \mathrm{C}^{\circ}$

[31] R. Castañeda, Pure and Appl. Opt. 3, p. 737 (1994)

[32] C. Preza et al., J. Opt. Soc. Am. A, 10, p. 1078 (1992)

[33] C. Preza et al., SPIE 1905, p. 129 (1993) 


\section{FIGURE CAPTIONS}

Fig. 1: (a) experimental noisy PSF. (b) diffraction limited computed PSF assuming design parameters only. The Look Up Table has been chosen so as to enhance the low level parts of the PSF and the noise.

Fig. 2: Method for identifying unknown PSF parameters by comparison of a reference PSF (experimental or simulated) with computed PSFs. The Gibson and Lanni model is used. Computed PSFs are centered in their domain of definition, as the reference PSF.

Fig. 3: Correlation curves between a computed reference PSF as function of one unknown parameter (a): numerical aperture of the objective, (b): objective immersion oil refractive index, and (c): depth of the specimen under the coverslip.

Fig. 4: Search for (a) : oil refractive index and (b) : specimen depth under the coverslip for an experimental PSF. The found values do correspond to the ones expected from the sample preparation and the acquisition procedure. The curves represent the quadratic error between the experimental denoised PSF and the computed PSFs.

Fig. 5: Deconvolution of images with correct and incorrect PSFs when simulating the use of the microscope at $37^{\circ} \mathrm{C}$. (a) Original $680 \mathrm{~nm}$ bead. (b) Correct aberrated $\mathrm{PSF}_{1.512}$ because of the oil refractive index drift induced by a temperature of $37^{\circ} \mathrm{C}$. (c) Simulated image. (d) Object reconstruction with PSF $_{1.512}$. (e) Object reconstruction with $\mathrm{PSF}_{1.515}$. (f) Incorrect $\mathrm{PSF}_{1.515}$ computed at $21^{\circ} \mathrm{C}$ assuming design parameters only. 


\section{TABLE CAPTION}

Table 1: results of a two-parameter identification on the numerical aperture and the depth of the specimen. 
Table 1:

\begin{tabular}{|c|c|c|}
\hline & numerical aperture & specthick $(\mu \mathrm{m})$ \\
\hline theoretical & 1.33 & 1.9 \\
\hline found & 1.325 & 1.1 \\
\hline
\end{tabular}




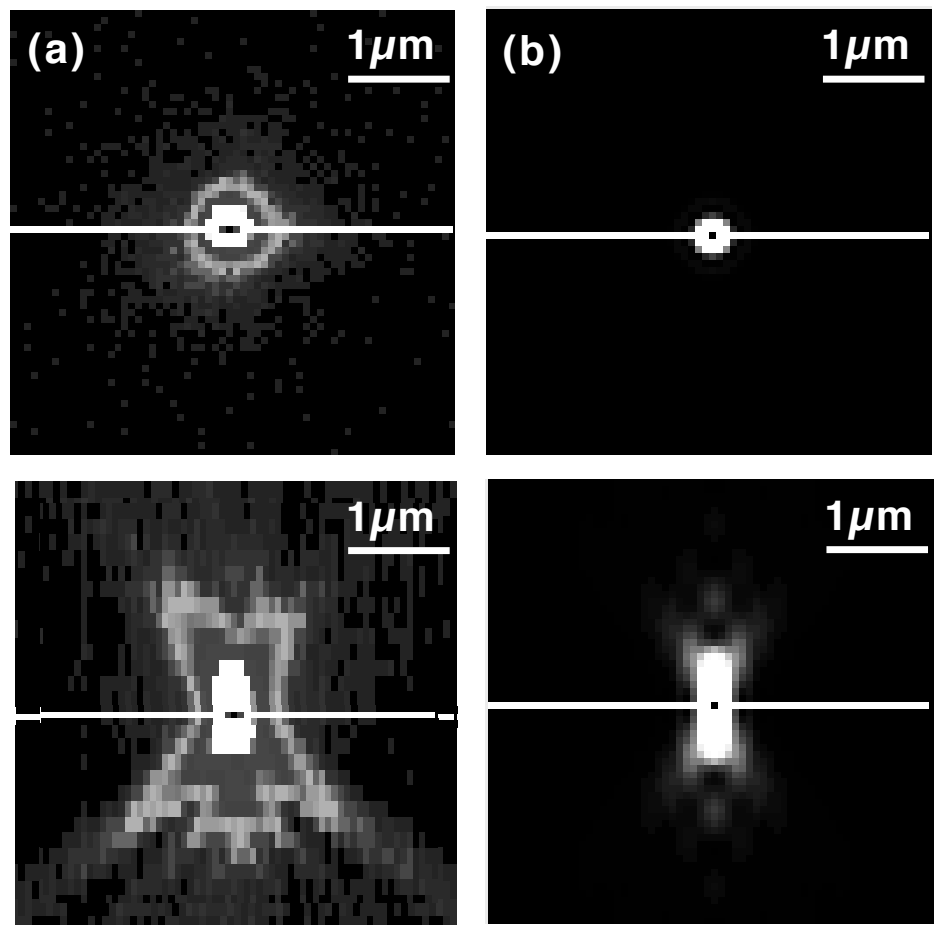

O. Haeberlé et al., "Identification of......." Figure 1 


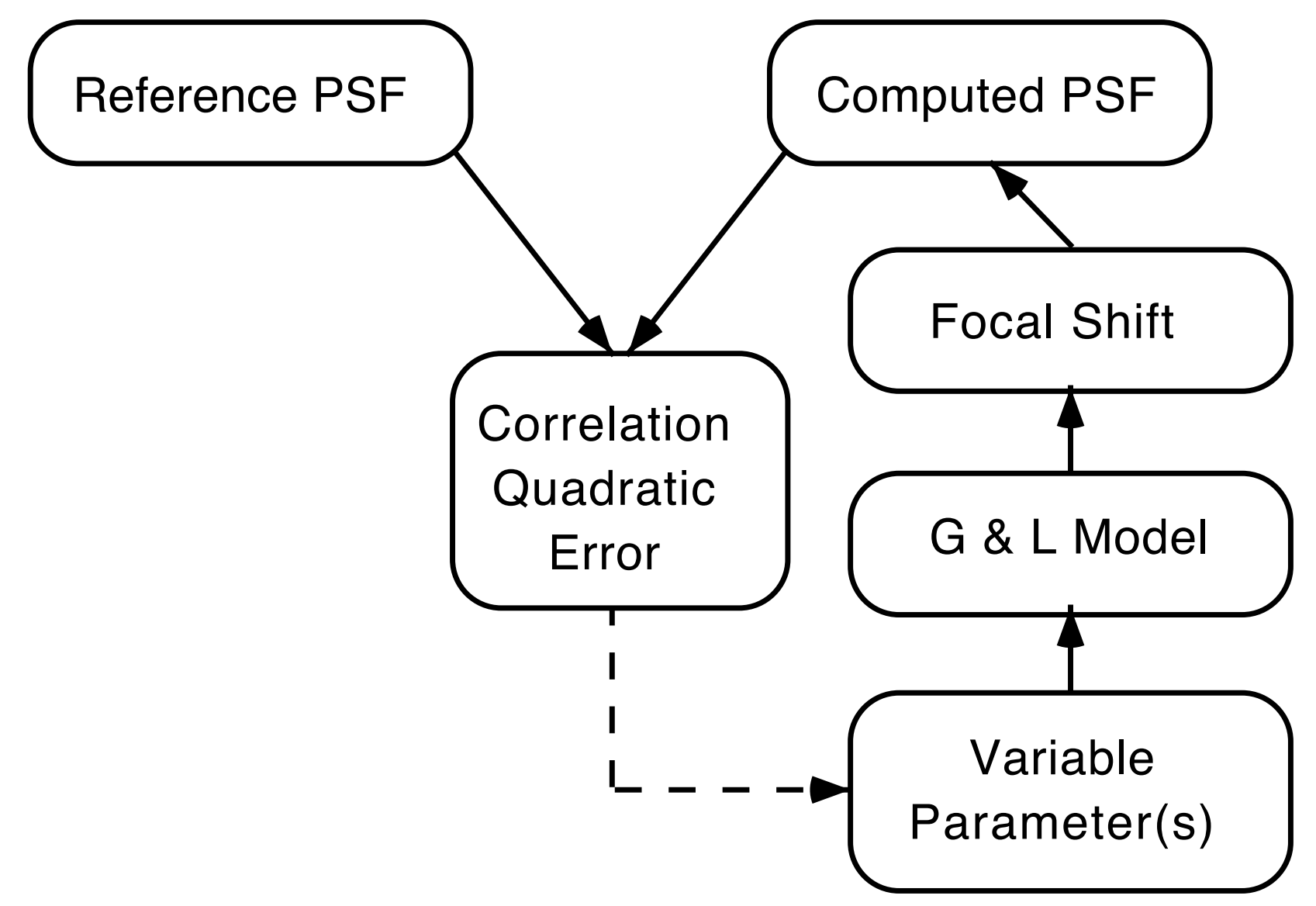




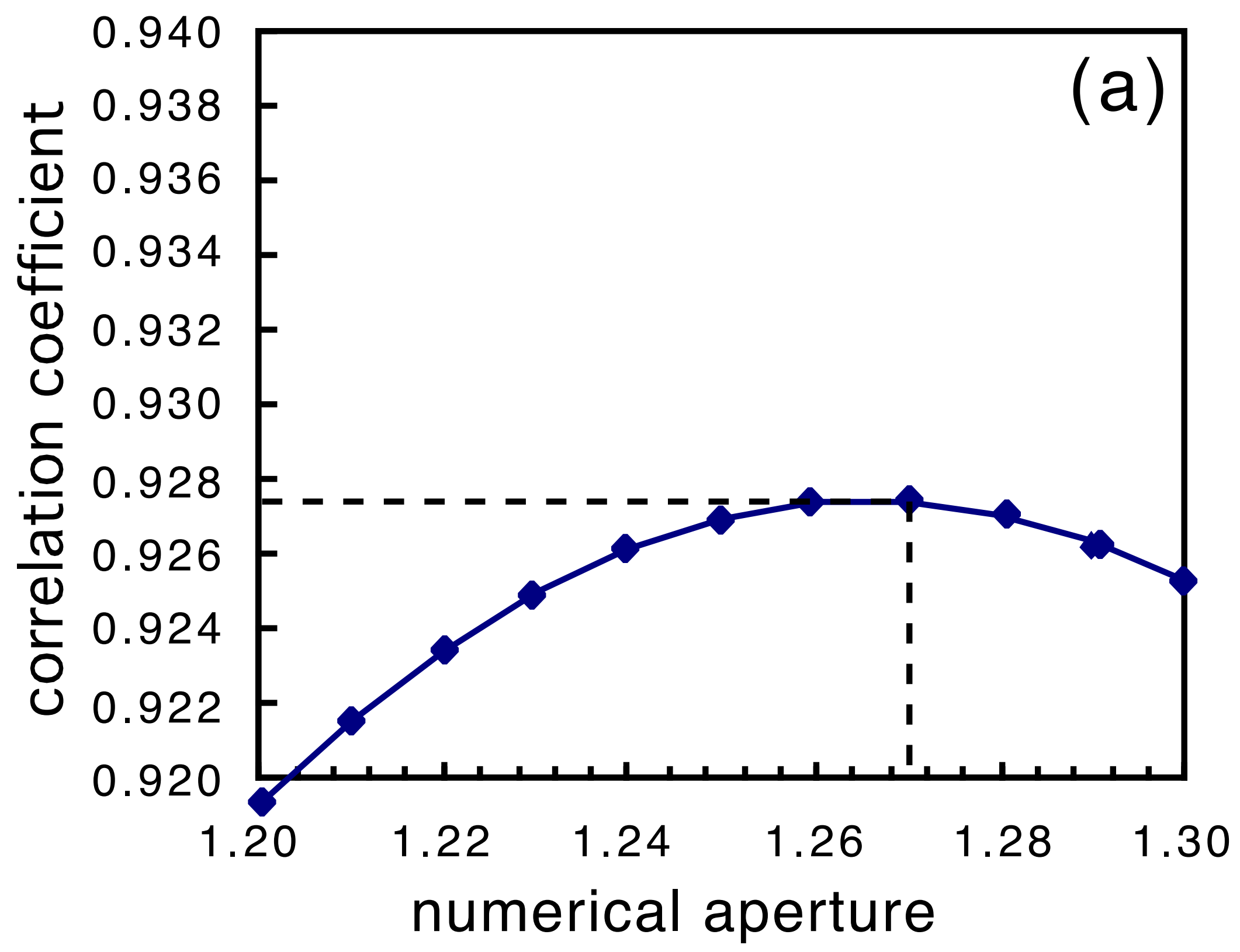




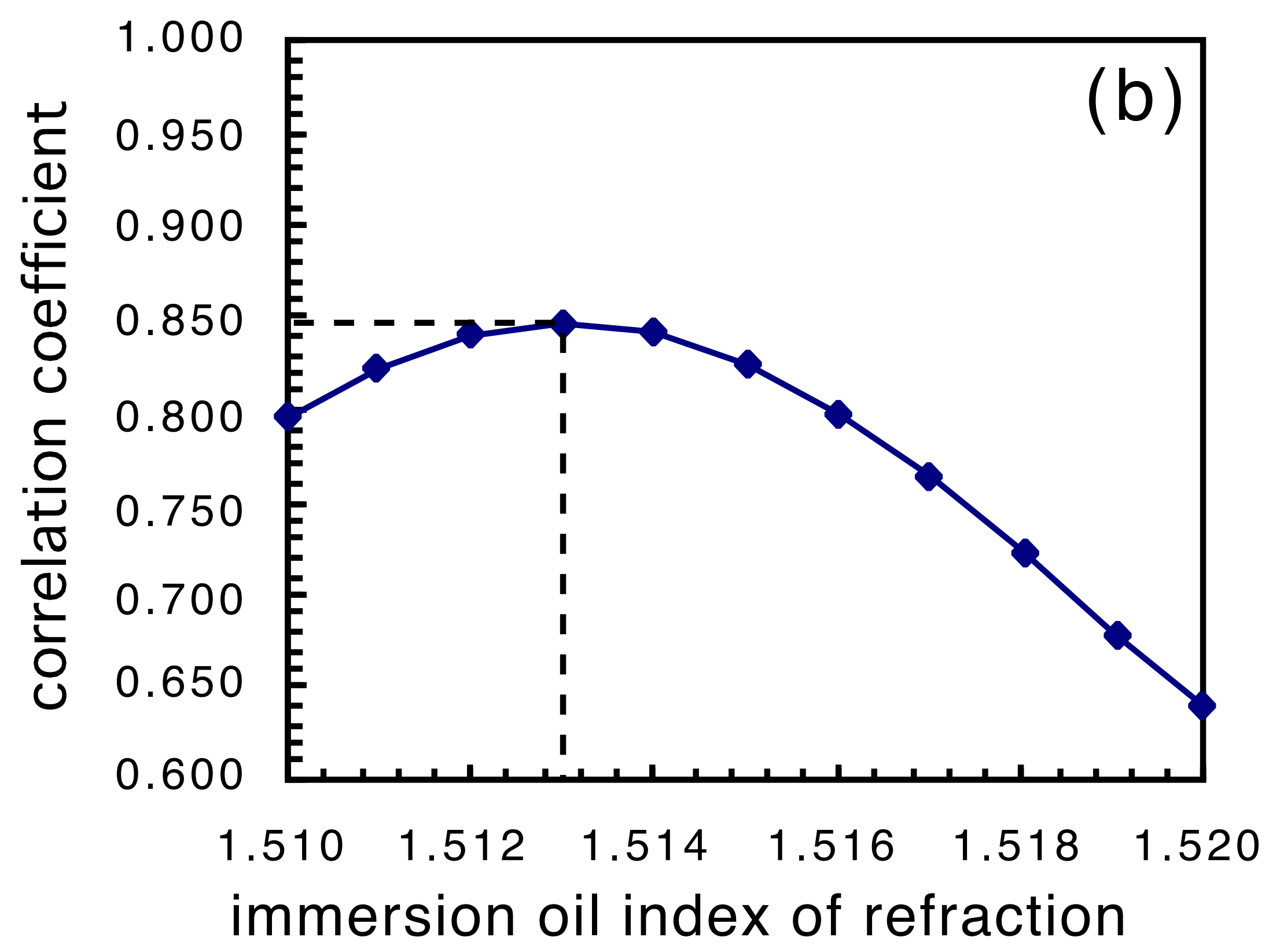




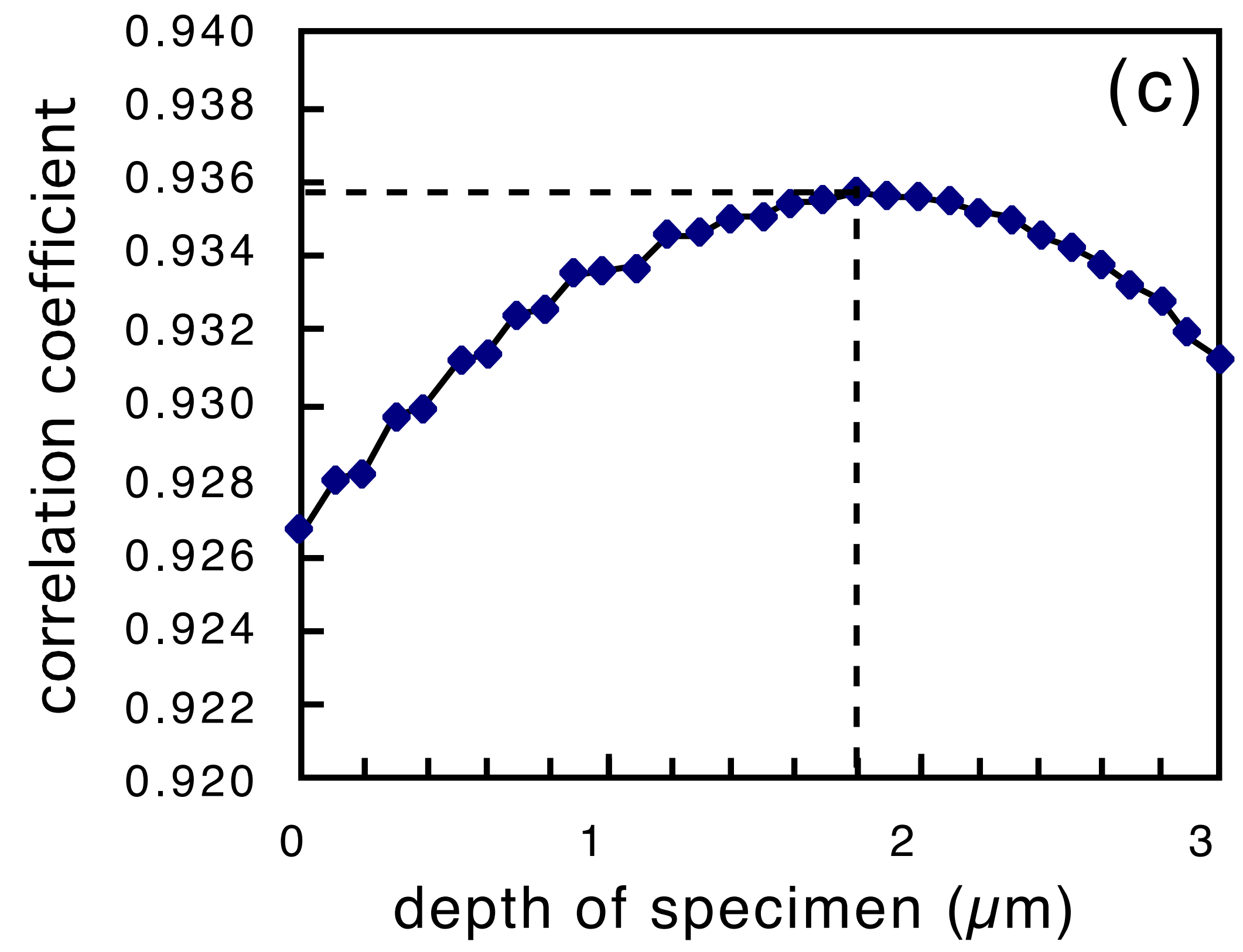




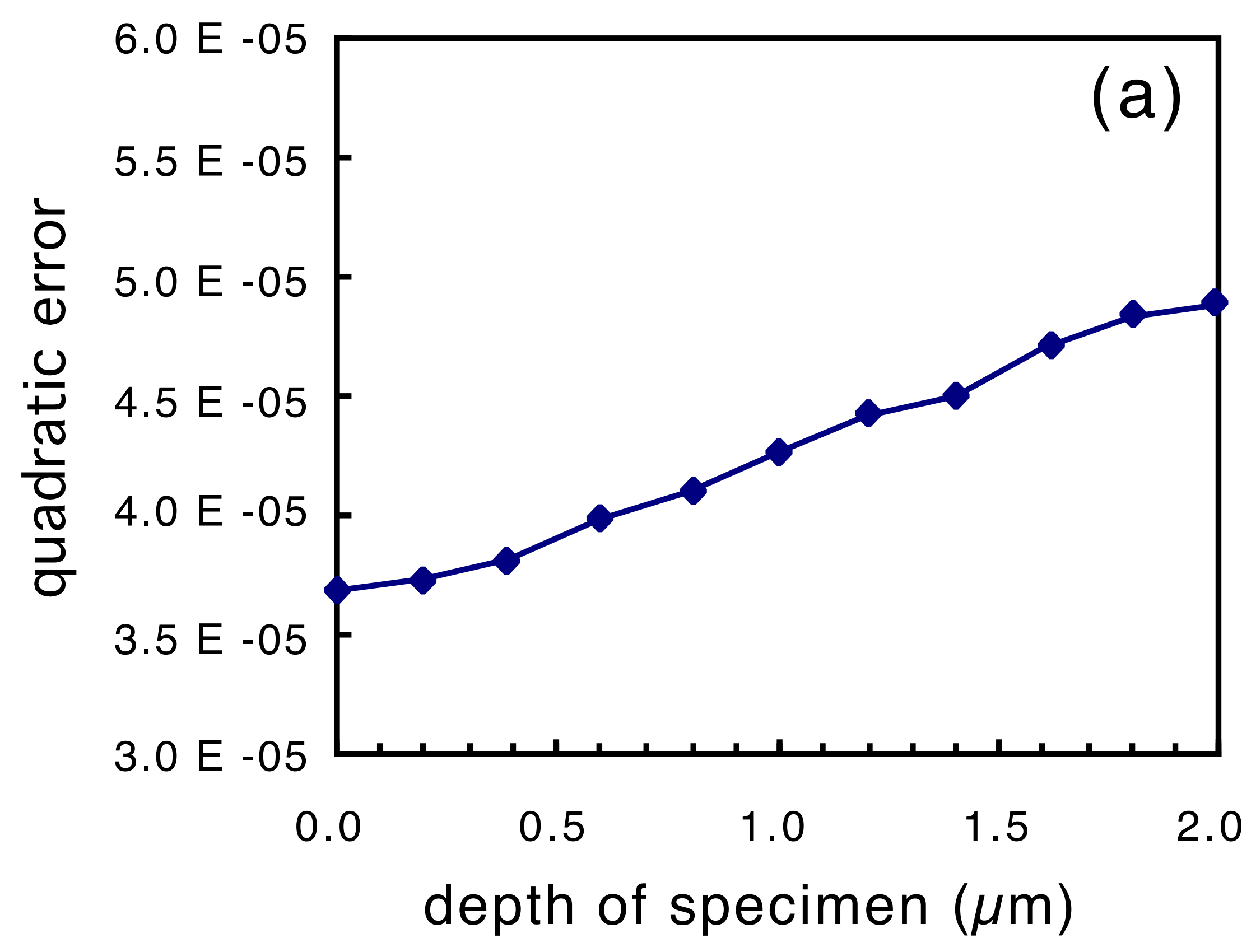




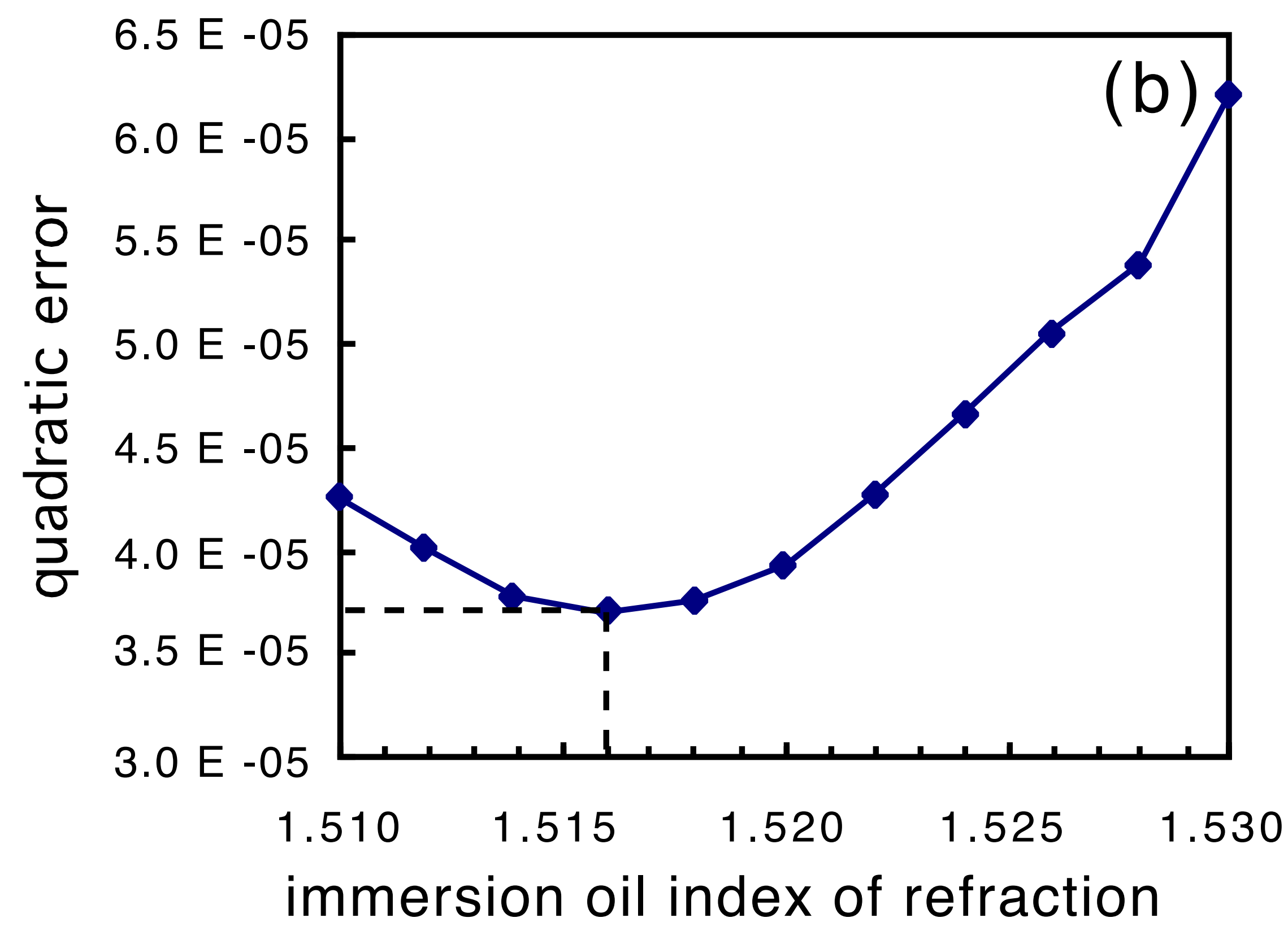




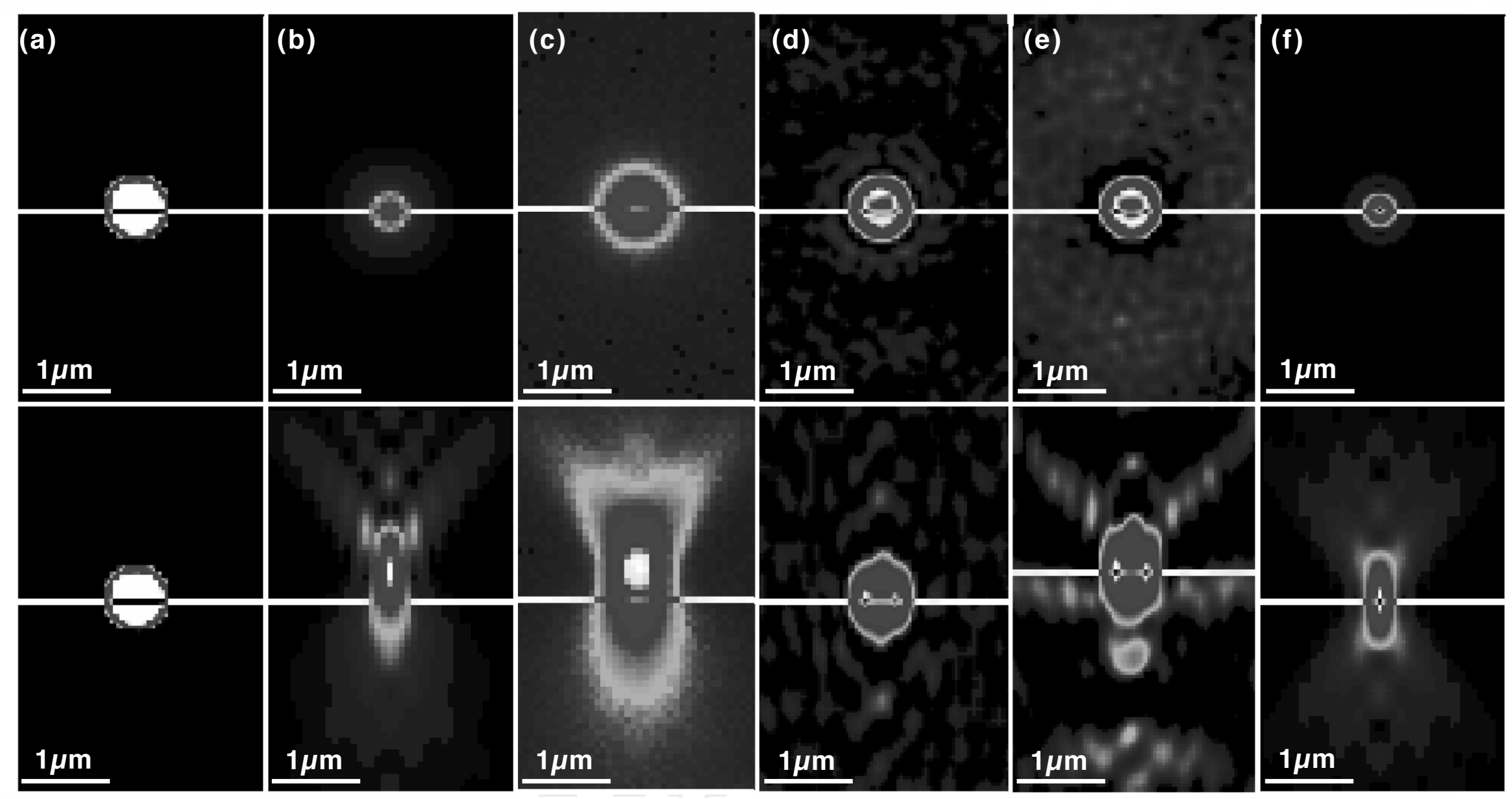

O. Haeberlé et al., "Identification of......." Figure 5 